\title{
ESCLEROSE MÚLTIPLA
}

\section{ANÁLISE CLÍNICA E EVOLUTIVA DE 214 CASOS}

\author{
CHARLES PETER TILBERY*, EDUARDO FELIPE* ${ }^{\star}$, CRISTINE MELLA BALDAUF* * \\ MÁRIO FERNANDO P. PERES***
}

\begin{abstract}
RESUMO - Os autores apresentam uma série de 214 casos de esclerose múltipla provenientes de levantamento retrospectivo de prontuários na Disciplina de Neurologia da Faculdade de Ciências Médicas da Santa Casa de São Paulo e da clínica privada, no período de 1980 a 1993 . Os dados encontrados foram analisados quanto a sexo, raça, média de idade de início dos sintomas, sintomas iniciais e sintomas evolutivos da doença. Os resultados foram analisados comparativamente aos encontrados em outras séries nacionais e estrangeiras.
\end{abstract}

PALAVRAS-CHAVE: esclerose múltipla, casuísticas nacionais, casuísticas estrangeiras.

\section{Multiple sclerosis: clinical and evolutive study of 214 cases}

SUMMARY - The authors report on a series of 214 cases of defined multiple sclerosis according to Poser et al. criteria. These cases were retrospectively selected by medical reports analysed from Santa Casa Medical School (São Paulo) and the private practive, from 1980 to 1993. The data were analysed as for sex, race, onset age of symptoms, onset symptoms, and evolutive symptoms. The results were compared to those found in other Brazilian series and in foreign series. This comparative analysis allows to verify similarities and differences among the several series, and the authors emphasize the necessity of multicentric studies in Brazil to assert with more details the multiple sclerosis profile in our country.

KEY-WORDS: multiple sclerosis, national (Brazilian) series, foreign series.

A esclerose múltipla (EM) é a mais frequente das doenças desmielinizantes ${ }^{2,13}$. A maioria dos autores admite uma distribuição geográfica típica da doença, com maior incidência em países de clima frio ou temperado e menor, em países de clima tropical ${ }^{8.9}$. No Brasil, a EM ainda é insuficientemente conhecida. Carecemos de estudos multicêntricos, que abordem os vários aspectos da doença. Há dificuldades inerentes ao diagnóstico que, até recentemente, baseava-se exclusivamente em critérios clínicos ${ }^{5}$. Contribui ainda para este fato, a falta de colaboração de colegas no registro de $\operatorname{casos}^{11}$ e a precariedade da assistência médica estatal ${ }^{5}$.

Nosso intuito, ao publicar esta casuistica, é contribuir para o conhecimento da EM em nosso País.

\section{MATERIAL E METÓDOS}

A presente série é constituída de 214 casos de EM examinados por um de nós (CPT) na clínica privada e por levantamento retrospectivo de prontuários na Disciplina de Neurologia da Faculdade de Ciências Médicas da Santa Casa de São Paulo, no período de 1980 a 1993.

Setor de Investigações em Neuroimunologia da Disciplina de Neurologia da Faculdade de Ciências Médicas da Santa Casa de São Paulo: *Professor Adjunto; **Instrutor de Ensino; ***Residente de terceiro ano; ****Acadêmico de medicina. Aceite: 5-dezembro-1994.

Dr. Charles Peter Tilbery - Rua Sampaio Vidal 855 - 01443-001 São Paulo SP - Brasil. 
Tabela 1. Frequência (\%) comparativa dos sintomas iniciais em 214 pacientes com EM em relação a outras séries estrangeiras.

\begin{tabular}{lcccc}
\hline Sintomas e sinais & Tilbery e col. & Kurtzke e col. $^{13}$ & Bauer $^{{ }^{3}}$ & Kuroiwa $^{{ }^{12}}$ \\
\hline \hline Piramidais & 47 & 54 & 43 & 24 \\
Tronco/Cerebelo & 32 & 42 & 22 & 34 \\
Ópticos & 27 & 25 & 36 & 42 \\
Sensitivos & 27 & 43 & 41 & 44 \\
Medulares & 12 & 30 & 10 & 38 \\
\hline
\end{tabular}

Tabela 2. Frequência (\%) comparativa dos sintomas iniciais em 214 pacientes com EM em relação a outras séries nacionais.

\begin{tabular}{lcccc}
\hline Sintomas e sinais & Tilbery e col. & Lana e Lana $^{14}$ & Callegaro $^{5}$ & Leite e col. $^{17}$ \\
\hline \hline Piramidais & 47 & 51 & 42 & 54 \\
Tronco/Cerebelo & 32 & 34 & 23 & - \\
Opticos & 27 & 43 & 17 & 35 \\
Sensitivos & 27 & 39 & 32 & 41 \\
Medulares & 12 & 22 & 15 & 29 \\
\hline
\end{tabular}

Foram selecionados pacientes que, segundo critérios estabelecidos por Poser e col. ${ }^{20}$, apresentavam quadro clínico compatível à forma definida de EM. Para tal confirmação diagnóstica utilizamos, além do exame neurológico, o exame de ressonância magnética da cabeça em 113 casos, o resultado do exame do liquído cefalorraqueano em 181 pacientes e o exame dos potenciais evocados em 107.

Os casos foram analisados considerando-se sexo, idade de início dos sintomas e raça dos pacientes. Os sintomas iniciais e do decurso da doença foram comparados aos da literatura estrangeira ocidental $^{3,12} \mathrm{e}$ oriental $^{11}$, bem como aos da literatura nacional ${ }^{5,14,17,19}$.

\section{RESULTADOS}

Dos 214 casos de EM da presente série, 175 apresentavam a forma remitente da doença (82\%) e 39 a forma progressiva primária (18\%).

$\mathrm{Na}$ forma remitente, 130 eram do sexo feminino e 45 do sexo masculino, com frequência de 1:2,8 homens/mulheres. Na forma progressiva primária, 29 pacientes eram do sexo feminino e 10 do masculino, com frequência de $1: 2,9$ homens/mulheres.

A média de idade do início dos sintomas no sexo feminino foi 28 anos na forma remitente e 36 anos na forma progressiva, enquanto no sexo masculino foi 30 anos de forma remitente e 28 anos na forma progressiva.

Em relação à raça, 166 pacientes eram brancos $(94 \%)$ e 9 eram negros $(5 \%)$ na forma remitente. Quanto à forma progressiva, 38 pacientes eram brancos (97\%) e 1 raça amarela (2\%).

A análise da frequência dos sintomas iniciais na EM da nossa casuística, comparativamente à estrangeiras, revela: semelhança quanto a série de Kurtzke ${ }^{13}$, para sintomas piramidais e visuais; com a de Bauer ${ }^{3}$, para sintomas piramidais e medulares; e com a de Kuroiwa ${ }^{12}$, nos itens relativos ao tronco cerebral e cerebelo. Nos demais, há diferenças, com ênfase maior para a baixa frequência, em relação a todas as séries, de sintomas sensitivos na nossa casuística (Tabela 1). 
Tabela 3. Frequência (\%) comparativa dos sintomas evolutivos em 214 pacientes com EM em relação a outras séries estrangeiras.

\begin{tabular}{lcccc}
\hline Sintomas e sinais & Tilbery e col. & Kurtzke e col. $^{13}$ & Bauer $^{3}$ & Kuroiwa $^{12}$ \\
\hline \hline Piramidais & 78 & 63 & 77 & 78 \\
Tronco/Cerebelo & 52 & 77 & 75 & 43 \\
Ópticos & 60 & 34 & 52 & 79 \\
Sensitivos & 67 & 55 & 73 & 75 \\
Medulares & 48 & 23 & 56 & 38 \\
\hline
\end{tabular}

Tabela 4. Frequência (\%) comparativa dos sintomas iniciais em 214 pacientes com EM em relação a outras séries nacionais.

\begin{tabular}{lccc}
\hline Sintomas e sinais & Tilbery e col. & Lana e Lana $^{14}$ & Callegaro $^{5}$ \\
\hline \hline Piramidais & 78 & 79 & 96 \\
Tronco/Cerebelo & 52 & 54 & 76 \\
Ópticos & 60 & 84 & 58 \\
Sensitivos & 67 & 90 & 71 \\
Medulares & 48 & 49 & 58 \\
\hline
\end{tabular}

Comparativamente às casuísticas nacionais, notam-se semelhanças entre as frequências de sintomas piramidais, de tronco e cerebelo. Para os demais sintomas, há diferenças; porém, nossa casuística assemelha-se à de Callegaros, exceção feita ao comprometimento visual (Tabela 2).

Ao comparar as frequências do comprometimento neurológico durante a evolução da doença da nossa série com as estrangeiras, nota-se semelhança com as séries de: Bauer ${ }^{3} \mathrm{e}$ Kuroiwa ${ }^{12}$, exceção feita a sintomas de tronco e visuais (Tabela 3 ).

Da mesma forma, a comparação dos sintomas evolutivos da nossa série mostra semelhanças com as séries nacionais de Lana e Lana ${ }^{14} \mathrm{e}$ Callegaros. Porém, há maior número de sintomas de comprometimento piramidal de tronco cerebral e cerebelo na casuística de Callegaro ${ }^{5}$ de sintomas visuais e sensitivos na série de Lana e Lana ${ }^{14}$ (Tabela 4).

\section{COMENTÁRIOS}

A nossa série apresenta imperfeições. A maioria dos casos foi analisada por estudo retrospectivo. Este procedimento não nos permitiu determinar, com o devido rigor, quais pacientes apresentavam a forma secundariamente progressiva, pois muitos pacientes não retornaram a todas as consultas de controle de evolução. Este fato também não nos permitiu determinar a frequência e duração dos surtos e remissões nas formas remitentes da doença, como fez Callegaro em sua série.

Os dados passíveis de análise e comparação, na nossa casuística, foram pareados com os dados disponíveis na literatura estrangeira e nacional. É importante salientar, por outro lado, que na série japonesa de Kuroiwa ${ }^{12}$, originariamente constituída de 1084 pacientes, procuramos analisar apenas os dados relativos a 509 pacientes, pois os restantes não eram da forma definida de EM (82 casos de doença de Devic e 357 de EM possível). 
Ao compararmos nossa série com a de Callegaros, notamos que a frquência das formas remitentes é semelhante (na nossa série $82 \%$, na de Callegaro ${ }^{5} 85 \%$ ). Outros autores relatam frequências variadas (Bernardi e col. ${ }^{4} 53,7 \%$; Lauer e col. ${ }^{15} 44,6 \%$; Leite e col. ${ }^{17} 47 \%$ ). A forma clínica primariamente progressiva na nossa casuística (18\%) é semelhante à de Leite e col. ${ }^{17}(19,7 \%)$ e de Lauer e col. ${ }^{15}(18 \%)$. Callegaro ${ }^{5}$ relata $10,2 \%$ dos seus casos como primariamente progressivos e Bernardi e col. $4,44,7 \%$.

O predomínio da doença no sexo feminino é relatado por todos os autores, havendo apenas diferenças de incidência de uma série para outra. Lana e Lana ${ }^{14}$ encontraram 1:2,3; Callegaro ${ }^{5}$ 1:1,6; Kuroiwa ${ }^{12} 1: 1,3$; Visscher e col. ${ }^{22} 1: 2,4 ; \operatorname{Tan}^{21} 1: 5$ e Leite e col. ${ }^{17} 1: 2,1$.

A média de idade de início dos sintomas de EM também é semelhante nas várias casuísticas. Leite e col. ${ }^{17}$ encontraram 31,4 anos, Lana e Lana ${ }^{14} 28,9$ anos, sem diferenciar, entretanto, as formas remitentes das progressivas e sem citar o sexo dos pacientes. Callegaro ${ }^{5}$ relata início dos sintomas, nas formas remitentes, na média de idade de 26,5 anos, sem, entretanto, especificar também o sexo dos pacientes. Este dado é semelhante ao nosso, apenas para pacientes do sexo feminino (na nossa série, a média de início para pacientes do sexo masculino foi 30 anos). Nas formas primariamente progressivas, Callegaro ${ }^{5}$ refere média de idade de início dos sintomas aos 36 anos (na nossa casuística este dado ocorreu apenas nas pacientes do sexo feminino) Não observamos na literatura dado comparável ao nosso para pacientes do sexo masculino, com formas progressivas primárias, nos quais a média de idade de início foi 28 anos. Há, na verdade, dados contraditórios na literatura neste aspecto $^{1,7,13}$.

O início da doença após os 40 anos de idade ocorreu em 32 casos da nossa série (15\%), dos quais $25(78 \%)$ eram do sexo feminino e 7 do masculino (21\%), como relatam também Callegaro ${ }^{5}$, Leibowitz ${ }^{16}$ e Confraveux e col.?.

A relação da EM com a raça dos pacientes é difícil de ser analisada, pois poucas são as regiōes no mundo onde há coexistência de várias raças. Entretanto, Callegaro ${ }^{5}$ relata na sua série $70 \%$ de pacientes brancos, $10 \%$ de pacientes pardos, $10 \%$ de pacientes negros e $8 \%$ de amarelos. Papaiz-Alvarenga e col. ${ }^{18}$ apresentam na sua casuística $62 \%$ de pacientes da raça branca e $29 \%$ da raça negra. Leite e col. ${ }^{17}$ referem apenas que $62,7 \%$ dos pacientes eram da raça branca. Na nossa casuistica houve nítido predomínio de pacientes de raça branca.

A comparação das séries publicadas envolve dificuldades inerentes aos dados passíveis desta confrontaçāo, pois nāo há padronização na coleta destes dados. Nota-se, claramente, que há diferenças e semelhanças entre todas elas. O predomínio de sinais motores piramidais como sintoma inicial em todas as séries nacionais ${ }^{5.14 .17 .19} \mathrm{e}$ ocidentais ${ }^{3,14}$ merece ser ressaltado. Na sćrie oriental japonesa ${ }^{12}$ este predomínio é de sintomas sensitivos e visuais. Analisando as séries nacionais, nota-se que sintomas iniciais de tronco e cerebelo são habituais em todas elas. O comprometimento visual nas séries é bastante diferente; porém, a série japonesa ${ }^{12}$ e a de Lana e Lana ${ }^{14}$ são semelhantes nesse aspecto, o que motivou Lana e Lana a sugerirem que a EM em nosso meio fosse do tipo oriental ${ }^{14}$. Sintomas iniciais sensitivos e medulares também são diferentes nas várias séries. A análise dos sintomas evolutivos na EM guarda, também, estas semelhanças e diferenças.

Ao analisar a nossa série e as casuísticas de Lana e Lana $^{14}$ e Callegaro ${ }^{5}$, nota-se que a Lana e Lana tem sintomas visuais e sensitivos predominantes e superiores às duas outras séries, na evolução da EM. Callegaro", por sua vez, apresenta, na sua série, sintomas piramidais, de tronco e de cerebelo mais frequentes na evolução de seus pacientes comparativamente às outras séries.

Fatores genéticos, raciais, geográficos foram aventados a fim de justificar a distribuição e as manifestações clínicas da doença ${ }^{4-6,14}$. No nosso meio, apenas Callegaro ${ }^{6}$ estudou a prevalência da EM e, assim mesmo, somente na cidade de São Paulo. Torna-se, portanto, mister, realizar estudo multicêntrico sobre a EM no Brasil, para que estes aspectos sejam melhor conhecidos. 


\section{REFERÊNCIAS}

1. Allison RS. Survival in disseminated sclerosis: a clinical study of a series of cases first seen twenty years ago. Brain 1950, 73:103-115.

2. Alter M, Halpern L, Kurland L, Bornstein B, Leibowitz U, Silberstein J. Multiple sclerosis in Israel: prevalence among immigrants and native inhabitants. Arch Neurol 1962, 7:253-263.

3. Bauer HJ. Problems of symptomatic therapy of multiple sclerosis. Neurology 1978, 28:8-20.

4. Bernardi S, Buttinelli C, Grasso MG, Millefiomi E, Pace A, Prencipe M, Fieschi C. Evolution and severity markers in 233 multiple sclerosis patients. Riv Neurol 1987, 57:197-200.

5. Callegaro D. Contribuição do estudo clínico evolutivo da esclerose múltipla: análise de 120 pacientes. Tese de Doutorado, Faculdade de Medicina da Universidade de São Paulo. São Paulo 1989.

6. Callegaro D, Lolio CA, Radvany J, Tilbery CP, Mendonça RA, Melo ACP. Prevalence of multiple sclerosis in the city of São Paulo, Brazil, in 1990. Neuroepidemiology 1992, 11:11-14.

7. Confavreux C, Aimard G, Devic M. Course and prognosis of multiple sclerosis: computerized data processing of 349 patients. Brain 1980, 103:281-300.

8. Dean G. Epidemiology of multiple sclerosis. Neuroepidemiology 1984, 3:58-73.

9. Detels R, Visscher BR, Malmgren RM, Coulson AM, Lucia MV, Dudley JP. Evidence for lower susceptibility to multiple sclerosis in Japanese-Americans. Am J Epidemiol 1977, 106:303-310.

10. Detels R, Clark VA, Valdiviezo NL, Visscher BR, Malmgren RM, Dudley JP. Factors associated with a rapid course of multiple sclerosis. Arch Neurol 1982, 39:337-341.

11. Granieri E, Tola R, Paolino E, Rosati G, Carreras M, Moneti VC. The frequency of multiple sclerosis in Italy: a descriptive study in Fevrana. Ann Neurol 1985, 1:80-84.

12. Kuroiwa Y. Nationwide survery of MS in Japan. Neurology 1975, 25:845-851.

13. Kurtzke JF, Beebe GW, Dagler B, Auth TL, Kurland LT, Defzger MD. Studies on the natural history of multiple sclerosis: clinical and laboratory findings at first diagnoses. Acta Neurol Scand 1972, 48:19-46.

14. Lana-Peixoto MA, Lana-Peixoto MI. Is multiple sclerosis in Brazil and Asia alike? Arq Neuropsiquiatr 1992, 50:419-425.

15. Lauer K, Fimhaber W, Reining R, Leuchtweis B. Epidemiological investigations into multiple sclerosis in southern Hesse: I. Methodological problems and basic epidemiologic characteristics. Acta Neurol Scand 1984, 70:257-265.

16. Leibowitz V, Antonovski H, Katz R, Alter M. Does pregnancy increase the risk of multiple sclerosis? J Neurol Neurosurg Psychiatry 1967, 30:354-357.

17. Leite ACCB, Andrade C, Novis S. Esclerose múltipla no Rio de Janeiro: apresentação clínica em 51 casos. Arq Neuropsiquiatr 1990, 48 (Supl): 66 A.

18. Mackay RP, Hirano A. Forms of benign multiple sclerosis: report of two "clinical silent cases" discovery at autopsy. Arch Neurol 1967, 17:588-600.

19. Papaiz-Alvarenga RM, Santos CMM, Bemardini JSA, Camargo SMGG. Esclerose múltipla: perfil clínico e epidemiológico no Rio de Janeiro. Arq Neuropsiquiatr 1990, 48 (Supl): 63 A.

20. Poser CM, Paty DW, Scheinberg L, McDonald WI, Davis FA, Ebers GC, Johnson KP, Silberg DH, Tourtellote WW. New diagnostic criteria for multiple sclerosis: guidelines for research protocols. Ann Neurol 1983, 227-231.

21. Tan CT. Multiple sclerosis in Malasia. Arch Neurol 1988, 45:624-627.

22. Visscher BR, Liu KS, Clarck VA, Detels R, Malmgren RM, Dudley JP. Onset symptoms as predictors of mortality and disability in multiple sclerosis. Acta Neurol Scand 1984, 70:321-328. 